\title{
Pearson-Readhead Survey from Space
}

\author{
R.A. Preston ${ }^{1}$, M.L. Lister ${ }^{1}$, S. J. Tingay ${ }^{2}$, \\ B.G. Piner ${ }^{1}$, D.W. Murphy ${ }^{1}$, \\ D. L. Jones ${ }^{1}$, D.L. Meier ${ }^{1}$, T.J. Pearson ${ }^{3}$, A.C.S. Readhead ${ }^{3}$, \\ H. Hirabayashi ${ }^{4}$, H. Kobayashi ${ }^{5}$, M. Inoue ${ }^{6}$
}

\begin{abstract}
We are using the VSOP space VLBI mission to observe a complete sample of Pearson-Readhead survey sources at $4.8 \mathrm{GHz}$ to determine core brightness temperatures and pc-scale jet properties. To date we have imaged 27 of the 31 objects in our sample. Our preliminary results show that the majority of objects contain strong core components that remain unresolved on baselines of $30,000 \mathrm{~km}$. The brightness temperatures of several cores significantly exceed $10^{12} \mathrm{~K}$, which is indicative of highly relativistically beamed emission. We also find that core brightness temperature is correlated with intraday variability in compact AGNs.
\end{abstract}

\section{Introduction}

Ground-based VLBI imaging surveys have been of critical importance in determining the general morphological and dynamical properties of the nuclear regions of active galaxies. Studies of individual sources alone leave many questions unanswered, and can even provide a biased view of nuclear activity. The statistical results obtained from imaging surveys have been key in providing constraints and insights for theoretical models of nuclear jets and environments. The same can be true for space-based VLBI imaging surveys.

The VSOP imaging survey we are undertaking is aimed at studying the nuclear regions of a complete sample of active galaxies. In addition to exploring the menagerie of source morphologies and dynamics, we are addressing a number of issues aimed ultimately at elucidating the fundamental characteristics of galactic nuclear activity and the physical conditions in these regions. These include the brightness temperature $\left(\mathrm{T}_{b}\right)$ distribution of the nuclei and the inner jet components, jet Doppler factors, and curvature.

\section{The Sample}

The complete sample from which we have drawn our sources is the PearsonReadhead sample, which is defined by the following criteria: (1) $\delta>35^{\circ}$; (2)

\footnotetext{
${ }^{1}$ Jet Propulsion Laboratory, California Institute of Technology, USA; ${ }^{2}$ Australia Telescope National Facility, Narrabri, Australia; ${ }^{3}$ California Institute of Technology, Pasadena, USA; ${ }^{4}$ Institute of Space and Astronautical Science, Sagamihara, Japan; ${ }^{5}$ National Astronomical Observatory, Mitaka, Tokyo, Japan; ${ }^{6}$ Nobeyama Radio Observatory, Minamisaku, Nagano, Japan
} 
$|b|>10^{\circ}$; and total flux density at $5 \mathrm{GHz}>1.3 \mathrm{Jy}$ (Pearson \& Readhead 1988). This sample is ideal for a VSOP survey because the sources are strong, the VSOP $u v$ coverages are especially good above $+35^{\circ}$ declination, and multiepoch ground-based VLBI data and other existing supporting data on these sources exceeds that of any other possible sample. Based on the multi-epoch ground-based VLBI observations at $5 \mathrm{GHz}$ of this sample involving two of us (TJP and ACR), we have been able to carefully choose a complete subset of this sample having compact emission that is most likely to show fringes on VSOP baselines.

\section{Discussion}

To date we have imaged 27 of the 31 sources in the sample (Lister et al., in preparation). A significant increase in fine-scale detail is apparent in the VSOP images compared to the ground VLBI image obtained when the spacecraft data is omitted.

The fine-scale structure of nearly all of our sources is dominated by a bright, unresolved core component, as is revealed in plots of visibility amplitude versus $u v$ distance. The curves show a pronounced flattening at baselines greater than an earth diameter, instead of dropping smoothly to zero. The brightness temperatures of many of these core components exceed $10^{12} \mathrm{~K}$ in the source rest frame, or above the theoretical limits of $10^{11}$ to $10^{12} \mathrm{~K}$ (Readhead 1994, Kellermann \& Pauliny-Toth 1969). To achieve $T_{b}$ 's this high, the simplest explanation is to assume that the emission is highly relativistically beamed, although standard numerical MHD simulations of jet formation would be challenged to produce jets of sufficient speed if the theoretical limit of $T_{b}$ is close to the lower end of the predicted range.

A significant fraction of all compact radio sources exhibit rapid, low-level flux density variations on timescales less than a day. The sources in our sample that exhibit intraday variability (IDV) tend to have higher $T_{b}$ 's (Tingay et al., in press). This suggests a strong connection between IDV and relativistic beaming.

Acknowledgments. We gratefully acknowledge the VSOP Project, which is led by the Japanese Institute of Space and Astronautical Science in cooperation with many organizations and radio telescopes around the world. This research was performed in part at the Jet Propulsion Laboratory, California Institute of Technology, under contract to NASA.

\section{References}

Pearson, T. J. \& Readhead, A. C. S. 1988, ApJ, 328, 114

Readhead, A. C. S. 1994, ApJ, 426, 51

Kellermann, K. I. \& Pauliny-Toth, I. I. K. 1969, ApJ, 155, L7 